\title{
Fixed point results for rational contraction in function weighted dislocated quasi-metric spaces with an application
}

\author{
Abdullah Shoaib ${ }^{*}$ (D), Qasim Mahmood', Aqeel Shahzad', Mohd Salmi Md Noorani² and \\ Stojan Radenović ${ }^{3}$
}

Correspondence:

abdullahshoaib15@yahoo.com 1 Department of Mathematics and Statistics, Riphah International University, I-14, 44000 Islamabad, Pakistan

Full list of author information is available at the end of the article

\section{Springer}

\begin{abstract}
The objective of this article is to introduce function weighted $L-R$-complete dislocated quasi-metric spaces and to present fixed point results fulfilling generalized rational type F-contraction for a multivalued mapping in these spaces. A suitable example confirms our results. We also present an application for a generalized class of nonlinear integral equations. Our results generalize and extend the results of Karapınar et al. (IEEE Access 7:89026-89032, 2019).
\end{abstract}

MSC: 46S40;47H10;54H25

Keywords: Function weighted $L-R$-complete dislocated quasi-metric spaces; Fixed point; Set-valued mappings

\section{Introduction and preliminaries}

In functional analysis, fixed point theory plays a vital role in elaborating the problems. Fixed point results for the multivalued functions were first examined by Nadler [24]. The work of Nadler has been cited by many mathematicians and brings to the level of ultimate advancement, see $[6,25,33]$. Dislocated metric space [21] is one of the generalizations of metric spaces among several generalizations, and it has applications in logic programming semantics [10]. Hussain et al. [11] extended this concept to dislocated $b$-metric space and obtained results for weak contractions. On the other hand, Wilson [39] introduced the quasi-metric space by excluding the symmetric conditions in the definition of metric spaces. Several extensions of quasi-metric space have been made, and some fixed point theorems have been obtained, see [1, 9, 16, 18-20, 28, 31]. Shoaib et al. [35] established results for multivalued functions in a dislocated quasi-metric space, see also [8, 37]. Rational type, Kannan type, and Reich type contractions on multivalued functions in double controlled quasi-metric type spaces [34,36] have been introduced, and some fixed point theorems have been obtained. Another generalization of metric space, named function weighted metric space or F-metric space (see, [2-4, 22]), was defined by Jleli [13]. Recently, Panda et al. [29] defined extended F-metric space and discussed a solution for AtanganaBaleanu fractional and Lp-Fredholm integral equations. Karapınar et al. [17] gave the idea

(c) The Author(s) 2021. This article is licensed under a Creative Commons Attribution 4.0 International License, which permits use, sharing, adaptation, distribution and reproduction in any medium or format, as long as you give appropriate credit to the original author(s) and the source, provide a link to the Creative Commons licence, and indicate if changes were made. The images or other third party material in this article are included in the article's Creative Commons licence, unless indicated otherwise in a credit line to the material. If material is not included in the article's Creative Commons licence and your intended use is not permitted by statutory regulation or exceeds the permitted use, you will need to obtain permission directly from the copyright holder. To view a copy of this licence, visit http://creativecommons.org/licenses/by/4.0/. 
of a function weighted quasi-metric space and examined the presence of a fixed point of functions in function weighted bi-complete quasi-metric spaces. Different efforts have been made in the field of F-contraction mapping [38] to exhibit certain results on fixed points of multivalued mappings. Hussain et al. [12] introduced Suzuki-Wardowski type, Rasham et al. [30] established rational Ćirić type, and Sgroi et al. [32] defined HardyRoger type F-contraction mappings. Some applications were also discussed by them. For more results, see $[5,7,14,15,23,26,27]$. In this article, we introduce function weighted $L$ - $R$-complete dislocated quasi-metric spaces and obtain fixed point results for multivalued mappings satisfying generalized rational type F-contraction in such spaces without the second condition (F2) and the third condition (F3) imposed on Wardowski's function [38]. A suitable example and an application confirm our results. We start with some basic concepts.

Definition $1.1([17])$ A function $h:(0,+\infty) \rightarrow \mathbb{R}$ is said to be

(i) logarithmic-like, if:

$$
\begin{aligned}
& \text { for each sequence }\left\{\tau_{m}\right\} \subset(0,+\infty) \text { satisfies } \\
& \lim _{m \rightarrow+\infty} h\left(\tau_{m}\right)=-\infty \quad \text { if and only if } \lim _{m \rightarrow+\infty} \tau_{m}=0 .
\end{aligned}
$$

(ii) nondecreasing function, if:

$$
0<\sigma<\tau \text { implies } h(\sigma)<h(\tau) .
$$

Let $\gamma$ denote the set of all logarithmic-like nondecreasing functions.

Definition 1.2 ([13]) For a mapping $\delta: M \times M \rightarrow[0,+\infty)$, if a pair $(h, C) \in \gamma \times[0,+\infty)$ exists for all $u, v, w \in M$, we have
$\left(\Delta_{1}\right) \delta(u, w)=\delta(w, u)$
$\left(\Delta_{2}\right) \delta(u, w)=0$ if and only if $u=w$;
$\left(\Delta_{3}\right)$ For any $j \in \mathbb{N}, j \geq 2$, we have

$$
\delta(u, w)>0 \quad \text { implies } \quad h(\delta(u, w)) \leq h\left(\sum_{i=1}^{j-1} \delta\left(v_{i}, v_{i+1}\right)+C\right.
$$

for every $\left(v_{i}\right)_{i=1}^{j} \subset M$ with $\left(v_{1}, v_{j}\right)=(u, w)$. Then $\delta$ is called an $\mathcal{F}$-metric or a function weighted metric [17] and $(M, \delta)$ is known as an $\mathcal{F}$-metric space or a function weighted metric space. If we exclude the condition $\left(\Delta_{1}\right)$ from Definition 1.2, then $\left(M, \delta_{q}\right)$ represents a function weighted quasi-metric space [17].

Definition 1.3 Let $\left(M, \delta_{q}\right)$ be a function weighted quasi-metric space. If we replace $\left(\Delta_{2}\right)$ with $\delta_{q}(u, w)=0$ implies $u=w$, that is, $\delta_{q}(u, u)$ may not be equal to zero, then we say that $\delta_{q}$ is a function weighted dislocated quasi-metric on $M$. We will denote this new metric by $\delta_{d q}$. Furthermore, the couple $\left(M, \delta_{d q}\right)$ is called a function weighted dislocated quasi-metric space. Note that any function weighted quasi-metric space is also a function weighted dislocated quasi-metric space but the converse is not true in general. 
Definition 1.4 Let $\left(M, \delta_{d q}\right)$ be a function weighted dislocated quasi-metric space. A sequence $\left\{u_{t}\right\}$ in $M$ is

(i) left convergent to some $u \in M$ if and only if $\lim _{m \rightarrow+\infty} \delta_{d q}\left(u_{m}, u\right)=0$ or, for every $\varepsilon>0$, we have $\delta_{d q}\left(u_{m}, u\right)<\varepsilon$ for all $m \geq t_{\varepsilon}$, where $t_{\varepsilon}$ is some integer depending on $\varepsilon$.

(ii) right convergent to some $u \in M$ if and only if $\lim _{t \rightarrow+\infty} \delta_{d q}\left(u, u_{t}\right)=0$ or, for every $\varepsilon>0$, we have $\delta_{d q}\left(u, u_{t}\right)<\varepsilon$ for all $t \geq t_{\varepsilon}$, where $t_{\varepsilon}$ is some integer depending on $\varepsilon$.

(iii) The sequence $\left\{u_{t}\right\}$ is $L$-R-convergent if and only if it is both left and right convergent.

(iv) The sequence $\left\{u_{t}\right\}$ is bi-convergent to some $u \in M$ if and only if $\lim _{t \rightarrow+\infty} \delta_{d q}\left(u, u_{t}\right)=\lim _{t \rightarrow+\infty} \delta_{d q}\left(u_{t}, u\right)=0$.

Lemma 1.5 Every L-R-convergent sequence in a function weighted dislocated quasi-metric space is bi-convergent.

Definition 1.6 Let $\left(M, \delta_{d q}\right)$ be a function weighted dislocated quasi-metric space. A sequence $\left\{u_{t}\right\}$ in $M$ is

(i) left Cauchy if and only if $\lim _{\substack{t, m \rightarrow+\infty \\ t>m}} \delta_{d q}\left(u_{m}, u_{t}\right)=0$ or, for every $\varepsilon>0$, we have $\delta_{d q}\left(u_{m}, u_{t}\right)<\varepsilon$ for all $t>m \geq t_{\varepsilon}$, where $t_{\varepsilon}$ is some integer depending on $\varepsilon$.

(ii) right Cauchy if and only if $\lim _{\substack{t, m \rightarrow+\infty \\ m>t}} \delta_{d q}\left(u_{m}, u_{t}\right)=0$ or, for every $\varepsilon>0$, we have $\delta_{d q}\left(u_{m}, u_{t}\right)<\varepsilon$ for all $m>t \geq t_{\varepsilon}$, where $t_{\varepsilon}$ is some integer depending on $\varepsilon$.

(iii) The sequence $\left\{u_{t}\right\}$ is bi-Cauchy if and only if it is both left and right Cauchy.

Definition 1.7 Let $\left(M, \delta_{d q}\right)$ be a function weighted dislocated quasi-metric space. Then $\left(M, \delta_{d q}\right)$ is

(i) right-complete if and only if each right-Cauchy sequence in $M$ is bi-convergent to some $u \in M$.

(ii) left-complete if and only if each left-Cauchy sequence in $M$ is bi-convergent to some $u \in M$.

(iii) bi-complete (or dual complete) if and only if it is both right- and left-complete.

(iv) $L$ - $R$-complete if and only if for every bi-Cauchy in $M$ is $L$ - $R$-convergent to some $u \in M$.

Remark 1.8 Every right-complete, left-complete, and bi-complete function weighted dislocated quasi-metric space is $L-R$-complete, but the converse is not true in general, so it is better to prove results in $L-R$-complete function weighted dislocated quasi-metric space instead of right-complete or left-complete or bi-complete.

Definition 1.9 Let $Q$ be a nonempty subset in a function weighted dislocated quasimetric space $\left(M, \delta_{d q}\right)$, and let $u \in M$. An element $w_{0} \in Q$ is called the best approximation in $Q$ for $u$ if

$$
\begin{array}{ll}
\delta_{d q}(u, Q)=\delta_{d q}\left(u, w_{0}\right), & \text { where } \delta_{d q}(u, Q)=\inf _{w \in Q} \delta_{d q}(u, w), \\
\delta_{d q}(Q, u)=\delta_{d q}\left(w_{0}, u\right), & \text { where } \delta_{d q}(Q, u)=\inf _{w \in Q} \delta_{d q}(w, u) .
\end{array}
$$

If each $a \in M$ has at least one best approximation in $Q$, then $Q$ is called a proximinal set. The set of all closed proximinal subsets of $M$ is denoted by $P(M)$. 
Definition 1.10 The function $H_{\delta_{d q}}: P(M) \times P(M) \rightarrow[0,+\infty)$, defined by

$$
H_{\delta_{d q}}(G, H)=\max \left\{\sup _{g \in G} \delta_{d q}(g, H), \sup _{h \in H} \delta_{d q}(G, h)\right\}
$$

is called Hausdorff-Pompeiu function weighted dislocated quasi-metric on $P(M)$.

Lemma 1.11 Suppose that $\left(M, \delta_{d q}\right)$ is a function weighted dislocated quasi-metric. Let $\left(P(M), H_{\delta_{d q}}\right)$ be a function weighted Hausdorff-Pompeiu quasi-metric space on $P(M)$. Then, for all $G, F \in P(M)$ and for each $g \in G$, there exists $f_{g} \in F$ that satisfies $\delta_{d q}(g, F)=$ $\delta_{d q}\left(g, f_{g}\right)$, and then

$$
H_{\delta_{d q}}(G, F) \geq \delta_{d q}\left(g, f_{g}\right) .
$$

\section{Main results}

Let $\left(M, \delta_{d q}\right)$ be an $L$-R-complete function weighted dislocated quasi-metric, $a_{0} \in M$ and $S: M \rightarrow P(M)$ be the multivalued mapping on $M$. Let $a_{1} \in S a_{0}$ such that $\delta_{d q}\left(a_{0}, S a_{0}\right)=$ $\delta_{d q}\left(a_{0}, a_{1}\right)$ and $\delta_{d q}\left(S a_{0}, a_{0}\right)=\delta_{d q}\left(a_{1}, a_{0}\right)$. Now, for $a_{1} \in M$, there exists $a_{2} \in S a_{1}$ such that $\delta_{d q}\left(a_{1}, S a_{1}\right)=\delta_{d q}\left(a_{1}, a_{2}\right)$ and $\delta_{d q}\left(S a_{1}, a_{1}\right)=\delta_{d q}\left(a_{2}, a_{1}\right)$. Continuing this process, we construct a sequence $a_{n}$ of points in $M$ such that $a_{n+1} \in S a_{n}$, and $a_{n+2} \in S a_{n+1}$ with $\delta_{d q}\left(a_{n}, S a_{n}\right)=\delta_{d q}\left(a_{n}, a_{n+1}\right), \delta_{d q}\left(S a_{n}, a_{n}\right)=\delta_{d q}\left(a_{n+1}, a_{n}\right)$ and $\delta_{d q}\left(a_{n+1}, S a_{n+1}\right)=\delta_{d q}\left(a_{n+1}, a_{n+2}\right)$, $\delta_{d q}\left(S a_{n+1}, a_{n+1}\right)=\delta_{d q}\left(a_{n+2}, a_{n+1}\right)$. We denote this iterative sequence by $\left\{M S\left(a_{n}\right)\right\}$ and say that $\left\{M S\left(a_{n}\right)\right\}$ is a sequence in $M$ generated by $a_{0}$. Now, we announce our first new result in this paper.

Theorem 2.1 Suppose that $\left(M, \delta_{d q}\right)$ is an L-R-complete function weighted dislocated quasi-metric with respect to $(h, C) \in \gamma \times[0,+\infty)$. Let $S: M \rightarrow P(M)$ be a multivalued mapping, $\mathcal{F}:(0,+\infty) \rightarrow \mathbb{R}$ be a strictly increasing mapping, $\tau>0, \mu_{1}, \mu_{2}, \mu_{3}, \mu_{4} \geq 0$, $\eta_{1}=\frac{\mu_{1}+\mu_{2}}{1-\mu_{3}-\mu_{4}}<1$ and $\eta_{2}=\frac{\mu_{1}+\mu_{3}}{1-\mu_{2}-\mu_{4}}<1$ such that

$$
\begin{aligned}
\tau+ & \max \left\{\mathcal{F}\left(H_{\delta_{d q}}(S g, S w)\right), \mathcal{F}\left(H_{\delta_{d q}}(S w, S g)\right)\right\} \\
\leq & \min \left\{\mathcal{F}\left(\mu_{1} \delta_{d q}(g, w)+\mu_{2} \delta_{d q}(g, S g)+\mu_{3} \delta_{d q}(w, S w)+\mu_{4} \frac{\delta_{d q}(g, S g) . \delta_{d q}(w, S w)}{1+\delta_{d q}(g, w)}\right),\right. \\
& \left.\mathcal{F}\left(\mu_{1} \delta_{d q}(w, g)+\mu_{2} \delta_{d q}(S g, g)+\mu_{3} \delta_{d q}(S w, w)+\mu_{4} \frac{\delta_{d q}(S g, g) \cdot \delta_{d q}(S w, w)}{1+\delta_{d q}(w, g)}\right)\right\},
\end{aligned}
$$

whenever $\min \left\{H_{\delta_{d q}}(S g, S w), H_{\delta_{d q}}(S w, S g)\right\}>0, g, w \in\left\{M S\left(g_{t}\right)\right\} \cup\left\{z^{*}\right\}$, where $\left\{M S\left(g_{t}\right)\right\} \rightarrow z^{*}$. Then $z^{*}$ is the fixed point of $S$.

Proof Consider the sequence $\left\{M S\left(g_{t}\right)\right\}$. By using Lemma 1.11 and inequality (2.1), we have

$$
\begin{aligned}
\tau+\mathcal{F}\left(\delta_{d q}\left(g_{t+1}, g_{t+2}\right)\right) \leq & \tau+\mathcal{F}\left(H_{\delta_{d q}}\left(S g_{t}, S g_{t+1}\right)\right) \\
\leq & \mathcal{F}\left(\mu_{1} \delta_{d q}\left(g_{t}, g_{t+1}\right)+\mu_{2} \delta_{d q}\left(g_{t}, S g_{t}\right)+\mu_{3} \delta_{d q}\left(g_{t+1}, S g_{t+1}\right)\right. \\
& \left.+\mu_{4} \frac{\delta_{d q}\left(g_{t}, S g_{t}\right) . \delta_{d q}\left(g_{t+1}, S g_{t+1}\right)}{1+\delta_{d q}\left(g_{t}, g_{t+1}\right)}\right)
\end{aligned}
$$




$$
\begin{aligned}
\leq & \mathcal{F}\left(\mu_{1} \delta_{d q}\left(g_{t}, g_{t+1}\right)+\mu_{2} \delta_{d q}\left(g_{t}, g_{t+1}\right)+\mu_{3} \delta_{d q}\left(g_{t+1}, g_{t+2}\right)\right. \\
& \left.+\mu_{4} \frac{\delta_{d q}\left(g_{t}, g_{t+1}\right) \cdot \delta_{d q}\left(g_{t+1}, g_{t+2}\right)}{1+\delta_{d q}\left(g_{t}, g_{t+1}\right)}\right) \\
\leq & \mathcal{F}\left(\left(\mu_{1}+\mu_{2}\right) \delta_{d q}\left(g_{t}, g_{t+1}\right)+\left(\mu_{3}+\mu_{4}\right) \delta_{d q}\left(g_{t+1}, g_{t+2}\right)\right) .
\end{aligned}
$$

As $\tau>0$, we have

$$
\mathcal{F}\left(\delta_{d q}\left(g_{t+1}, g_{t+2}\right)\right)<\mathcal{F}\left(\left(\mu_{1}+\mu_{2}\right) \delta_{d q}\left(g_{t}, g_{t+1}\right)+\left(\mu_{3}+\mu_{4}\right) \delta_{d q}\left(g_{t+1}, g_{t+2}\right)\right) \text {. }
$$

As $\mathcal{F}$ is a strictly increasing mapping, we have

$$
\delta_{d q}\left(g_{t+1}, g_{t+2}\right)<\left(\mu_{1}+\mu_{2}\right) \delta_{d q}\left(g_{t}, g_{t+1}\right)+\left(\mu_{3}+\mu_{4}\right) \delta_{d q}\left(g_{t+1}, g_{t+2}\right) .
$$

We get

$$
\begin{aligned}
& \left(1-\mu_{3}-\mu_{4}\right) \delta_{d q}\left(g_{t+1}, g_{t+2}\right)<\left(\mu_{1}+\mu_{2}\right) \delta_{d q}\left(g_{t}, g_{t+1}\right), \\
& \delta_{d q}\left(g_{t+1}, g_{t+2}\right)<\left(\frac{\mu_{1}+\mu_{2}}{1-\mu_{3}-\mu_{4}}\right) \delta_{d q}\left(g_{t}, g_{t+1}\right) .
\end{aligned}
$$

As $\eta_{1}=\frac{\mu_{1}+\mu_{2}}{1-\mu_{3}-\mu_{4}}<1$, so

$$
\delta_{d q}\left(g_{t+1}, g_{t+2}\right)<\eta_{1} \delta_{d q}\left(g_{t}, g_{t+1}\right) .
$$

Let $\eta=\max \left\{\eta_{1}, \eta_{2}\right\}<1$, hence

$$
\delta_{d q}\left(g_{t+1}, g_{t+2}\right)<\eta \delta_{d q}\left(g_{t}, g_{t+1}\right) .
$$

Now, by using Lemma 1.11 and inequality (2.1), we have

$$
\begin{aligned}
\tau+\mathcal{F}\left(\delta_{d q}\left(g_{t}, g_{t+1}\right)\right) \leq & \tau+\mathcal{F}\left(H_{\delta_{d q}}\left(S g_{t-1}, S g_{t}\right)\right) \\
\leq & \mathcal{F}\left(\mu_{1} \delta_{d q}\left(g_{t-1}, g_{t}\right)+\mu_{2} \delta_{d q}\left(g_{t}, S g_{t}\right)+\mu_{3} \delta_{d q}\left(g_{t-1}, S g_{t-1}\right)\right. \\
& \left.+\mu_{4} \frac{\delta_{d q}\left(g_{t}, S g_{t}\right) \delta_{d q}\left(g_{t-1}, S g_{t-1}\right)}{1+\delta_{d q}\left(g_{t-1}, g_{t}\right)}\right) \\
\leq & \mathcal{F}\left(\mu_{1} \delta_{d q}\left(g_{t-1}, g_{t}\right)+\mu_{2} \delta_{d q}\left(g_{t}, g_{t+1}\right)+\mu_{3} \delta_{d q}\left(g_{t-1}, g_{t}\right)\right. \\
& \left.+\mu_{4} \frac{\delta_{d q}\left(g_{t-1}, g_{t}\right) . \delta_{d q}\left(g_{t}, g_{t+1}\right)}{1+\delta_{d q}\left(g_{t-1}, g_{t}\right)}\right) \\
\leq & \mathcal{F}\left(\left(\mu_{1}+\mu_{3}\right) \delta_{d q}\left(g_{t-1}, g_{t}\right)+\left(\mu_{2}+\mu_{4}\right) \delta_{d q}\left(g_{t}, g_{t+1}\right)\right)
\end{aligned}
$$

This implies

$$
\mathcal{F}\left(\delta_{d q}\left(g_{t}, g_{t+1}\right)\right)<\mathcal{F}\left(\left(\mu_{1}+\mu_{3}\right) \delta_{d q}\left(g_{t-1}, g_{t}\right)+\left(\mu_{2}+\mu_{4}\right) \delta_{d q}\left(g_{t}, g_{t+1}\right)\right) .
$$


Since $\mathcal{F}$ is a strictly increasing mapping, we have

$$
\delta_{d q}\left(g_{t}, g_{t+1}\right)<\left(\mu_{1}+\mu_{3}\right) \delta_{d q}\left(g_{t-1}, g_{t}\right)+\left(\mu_{2}+\mu_{4}\right) \delta_{d q}\left(g_{t}, g_{t+1}\right)
$$

We get

$$
\begin{aligned}
& \left(1-\mu_{2}-\mu_{4}\right) \delta_{d q}\left(g_{t}, g_{t+1}\right)<\left(\mu_{1}+\mu_{3}\right) \delta_{d q}\left(g_{t-1}, g_{t}\right), \\
& \delta_{d q}\left(g_{t}, g_{t+1}\right)<\left(\frac{\mu_{1}+\mu_{3}}{1-\mu_{2}-\mu_{4}}\right) \delta_{d q}\left(g_{t-1}, g_{t}\right) .
\end{aligned}
$$

As $\eta_{2}=\frac{\mu_{1}+\mu_{3}}{1-\mu_{2}-\mu_{4}}<1$, so

$$
\delta_{d q}\left(g_{t}, g_{t+1}\right)<\eta_{2} \delta_{d q}\left(g_{t-1}, g_{t}\right)<\eta \delta_{d q}\left(g_{t-1}, g_{t}\right)
$$

By using (2.3) in (2.2), we have

$$
\delta_{d q}\left(g_{t+1}, g_{t+2}\right)<\eta^{2} \delta_{d q}\left(g_{t-1}, g_{t}\right)
$$

Continuing in this way, we have

$$
\delta_{d q}\left(g_{t+1}, g_{t+2}\right)<\eta^{t+1} \delta_{d q}\left(g_{0}, g_{1}\right)
$$

By using Lemma 1.11 and inequality (2.1), we have

$$
\begin{aligned}
\tau+\mathcal{F}\left(\delta_{d q}\left(g_{t+2}, g_{t+1}\right)\right) \leq & \tau+\mathcal{F}\left(H_{\delta_{d q}}\left(S g_{t+1}, S g_{t}\right)\right) \\
\leq & \mathcal{F}\left(\mu_{1} \delta_{d q}\left(g_{t+1}, g_{t}\right)+\mu_{2} \delta_{d q}\left(S g_{t}, g_{t}\right)+\mu_{3} \delta_{d q}\left(S g_{t+1}, g_{t+1}\right)\right. \\
& \left.+\mu_{4} \frac{\delta_{d q}\left(S g_{t}, g_{t}\right) . \delta_{d q}\left(S g_{t+1}, g_{t+1}\right)}{1+\delta_{d q}\left(g_{t+1}, g_{t}\right)}\right) \\
\leq & \mathcal{F}\left(\mu_{1} \delta_{d q}\left(g_{t+1}, g_{t}\right)+\mu_{2} \delta_{d q}\left(g_{t+1}, g_{t}\right)+\mu_{3} \delta_{d q}\left(g_{t+2}, g_{t+1}\right)\right. \\
& \left.+\mu_{4} \frac{\delta_{d q}\left(g_{t+1}, g_{t}\right) \cdot \delta_{d q}\left(g_{t+2}, g_{t+1}\right)}{1+\delta_{d q}\left(g_{t+1}, g_{t}\right)}\right) \\
\leq & \mathcal{F}\left(\left(\mu_{1}+\mu_{2}\right) \delta_{d q}\left(g_{t+1}, g_{t}\right)+\left(\mu_{3}+\mu_{4}\right) \delta_{d q}\left(g_{t+2}, g_{t+1}\right)\right)
\end{aligned}
$$

Again by doing similar steps to obtain (2.2) from (2.1), we have

$$
\delta_{d q}\left(g_{t+2}, g_{t+1}\right)<\eta_{1} \delta_{d q}\left(g_{t+1}, g_{t}\right)<\eta \delta_{d q}\left(g_{t+1}, g_{t}\right) .
$$

By using Lemma 1.11 and inequality (2.1), we have

$$
\begin{aligned}
\tau+\mathcal{F}\left(\delta_{d q}\left(g_{t+1}, g_{t}\right)\right) & \leq \tau+\mathcal{F}\left(H_{\delta_{d q}}\left(S g_{t}, S g_{t-1}\right)\right) \\
& \leq \mathcal{F}\left(\mu_{1} \delta_{d q}\left(g_{t}, g_{t-1}\right)+\mu_{2} \delta_{d q}\left(S g_{t}, g_{t}\right)+\mu_{3} \delta_{d q}\left(S g_{t-1}, g_{t-1}\right)\right.
\end{aligned}
$$




$$
\begin{aligned}
& \left.+\mu_{4} \frac{\delta_{d q}\left(S g_{t}, g_{t}\right) \cdot \delta_{d q}\left(S g_{t-1}, g_{t-1}\right)}{1+\delta_{d q}\left(g_{t}, g_{t-1}\right)}\right) \\
\leq & \mathcal{F}\left(\mu_{1} \delta_{d q}\left(g_{t}, g_{t-1}\right)+\mu_{2} \delta_{d q}\left(g_{t+1}, g_{t}\right)+\mu_{3} \delta_{d q}\left(g_{t}, g_{t-1}\right)\right. \\
& \left.+\mu_{4} \frac{\delta_{d q}\left(g_{t+1}, g_{t}\right) \cdot \delta_{d q}\left(g_{t}, g_{t-1}\right)}{1+\delta_{d q}\left(g_{t}, g_{t-1}\right)}\right) \\
\leq & \mathcal{F}\left(\left(\mu_{1}+\mu_{3}\right) \delta_{d q}\left(g_{t}, g_{t-1}\right)+\left(\mu_{2}+\mu_{4}\right) \delta_{d q}\left(g_{t+1}, g_{t}\right)\right) .
\end{aligned}
$$

Again by doing similar steps to obtain (2.3) from (2.1), we have

$$
\delta_{d q}\left(g_{t+1}, g_{t}\right)<\eta_{2} \delta_{d q}\left(g_{t}, g_{t-1}\right)<\eta \delta_{d q}\left(g_{t}, g_{t-1}\right)
$$

By using (2.6) in (2.5), we have

$$
\delta_{d q}\left(g_{t+2}, g_{t+1}\right)<\eta^{2} \delta_{d q}\left(g_{t}, g_{t-1}\right) .
$$

Continuing in this way, we have

$$
\delta_{d q}\left(g_{t+2}, g_{t+1}\right)<\eta^{t+1} \delta_{d q}\left(g_{1}, g_{0}\right) .
$$

As $(h, C) \in \gamma \times[0,+\infty)$ satisfies $\left(\Delta_{3}\right)$, then for fixed $\epsilon>0$ there exists $\delta>0$ such that

$$
0<\sigma<\delta \text { implies } h(\sigma)<h(\epsilon)-C \text {. }
$$

By using (2.4), we have

$$
\begin{aligned}
& \sum_{k=n}^{m-1} \delta_{d q}\left(g_{k}, g_{k+1}\right)<\eta^{n}\left(1+\eta+\eta^{2} \ldots \eta^{m-n-1}\right) \delta_{d q}\left(g_{0}, g_{1}\right), \\
& \sum_{k=n}^{m-1} \delta_{d q}\left(g_{k}, g_{k+1}\right)<\frac{\eta^{n}}{1-\eta} \delta_{d q}\left(g_{0}, g_{1}\right), \quad m>n .
\end{aligned}
$$

Since $\lim _{n \rightarrow+\infty} \frac{\eta^{n}}{1-\eta} \delta_{d q}\left(g_{0}, g_{1}\right)=0$, then for $\delta>0$ there exists some $n_{0} \in \mathbb{N}$ such that $0<$ $\frac{\eta^{n}}{1-\eta} \delta_{d q}\left(g_{0}, g_{1}\right)<\delta, n \geq n_{0}$. By (2.8) and (2.9), we write

$$
\begin{aligned}
h\left(\sum_{k=n}^{m-1} \delta_{d q}\left(g_{k}, g_{k+1}\right)\right) & <h\left(\frac{\eta^{n}}{1-\eta} \delta_{d q}\left(g_{0}, g_{1}\right)\right) \\
& <h(\epsilon)-C \text { for all } m, n \geq n_{0} .
\end{aligned}
$$

Suppose that $\delta_{d q}\left(g_{p}, g_{d q}\right)=0$ for some $p, q \in\{0,1,2,3, \ldots\}$ with $q>p$, then $g_{p}=g_{d q}$

$$
\begin{aligned}
& \delta_{d q}\left(g_{p}, g_{p+1}\right)=\delta_{d q}\left(g_{p}, S g_{p}\right)=\delta_{d q}\left(g_{d q}, S g_{d q}\right)=\delta_{d q}\left(g_{d q}, g_{q+1}\right) \leq \eta^{q-p} \delta_{d q}\left(g_{p}, g_{p+1}\right), \\
& \left(1-\eta^{q-p}\right) \delta_{d q}\left(g_{p}, g_{p+1}\right) \leq 0 .
\end{aligned}
$$


So $\delta_{d q}\left(g_{p}, g_{p+1}\right)=0$ and $g_{p}=g_{p+1}$. Now, $g_{p+1} \in S g_{p}$ implies that $g_{p} \in S g_{p}$. Hence $g_{p}$ is the fixed point of $S$. Now suppose that $\delta_{d q}\left(g_{m}, g_{n}\right) \neq 0$ for all $m, n \in\{0,1,2,3, \ldots\}$ with $m>n$. Using $\left(\Delta_{3}\right)$ and the inequality, $\delta_{d q}\left(g_{n}, g_{m}\right)>0$ for all $m, n \geq n_{0}$, we have

$$
\begin{aligned}
& h\left(\delta_{d q}\left(g_{n}, g_{m}\right)\right)<h\left(\sum_{k=n}^{m-1} \delta_{d q}\left(g_{k}, g_{k+1}\right)\right)+C<h(\epsilon), \\
& \delta_{d q}\left(g_{n}, g_{m}\right)<\epsilon \quad \text { for all } m, n \geq n_{0} .
\end{aligned}
$$

This proves that $\left\{g_{n}\right\}$ is a right-Cauchy sequence in $M$. Again by using (2.7), we have

$$
\begin{aligned}
\sum_{k=n}^{m-1} \delta_{d q}\left(g_{k+1,} g_{k}\right) & \leq \eta^{n}\left(1+\eta+\eta^{2} \ldots \eta^{m-n-1}\right) \delta_{d q}\left(g_{1}, g_{0}\right) \\
& \leq \frac{\eta^{n}}{1-\eta} \delta_{d q}\left(g_{1}, g_{0}\right), \quad m>n .
\end{aligned}
$$

Since $\lim _{n \rightarrow+\infty} \frac{\eta^{n}}{1-\eta} \delta_{d q}\left(g_{1}, g_{0}\right)=0$, for any $\delta>0$ there exists some $n_{1} \in \mathbb{N}$ such that $0<$ $\frac{\eta^{n}}{1-\eta} \delta_{d q}\left(g_{1}, g_{0}\right)<\delta$ for all $n \geq n_{1}$. Furthermore, assume that $(h, C) \in \gamma \times[0,+\infty)$ satisfies $\left(\Delta_{3}\right)$, and let $\epsilon>0$ be fixed, by using similar steps as above, we have

$$
\delta_{d q}\left(g_{m}, g_{n}\right)<\epsilon \text { for all } m, n \geq n_{1} .
$$

This proves that $\left\{g_{n}\right\}$ is a left-Cauchy sequence in $M$. Hence, $\left\{g_{n}\right\}$ is a bi-Cauchy sequence in $M$. Since $\left(M, \delta_{d q}\right)$ is $L$ - $R$-complete, there will be some $y^{*} \in M$ such that $\left\{g_{n}\right\}$ is $L-R$ convergent to $y^{*}$. By Lemma 1.5, every $L-R$-convergent sequence is bi-convergent, that is,

$$
\lim _{t \rightarrow+\infty} \delta_{d q}\left(z^{*}, g_{t}\right)=\lim _{t \rightarrow+\infty} \delta_{d q}\left(g_{t}, z^{*}\right)=0
$$

Suppose $\delta_{d q}\left(z^{*}, S z^{*}\right)>0$, we have

$$
\begin{aligned}
\tau+\mathcal{F}\left(\delta_{d q}\left(g_{t+1}, S z^{*}\right)\right) \leq & \tau+\mathcal{F}\left(H_{\delta_{d q}}\left(S g_{t}, S z^{*}\right)\right) \\
\leq & \mathcal{F}\left(\mu_{1} \delta_{d q}\left(g_{t}, z^{*}\right)+\mu_{2} \delta_{d q}\left(g_{t}, S g_{t}\right)+\mu_{3} \delta_{d q}\left(z^{*}, S z^{*}\right)\right. \\
& \left.+\mu_{4} \frac{\delta_{d q}\left(g_{t}, S g_{t}\right) \cdot \delta_{d q}\left(z^{*}, S z^{*}\right)}{1+\delta_{d q}\left(g_{t}, z^{*}\right)}\right) .
\end{aligned}
$$

This implies that

$$
\begin{aligned}
\delta_{d q}\left(g_{t+1}, S z^{*}\right)< & \mu_{1} \delta_{d q}\left(g_{t}, z^{*}\right)+\mu_{2} \delta_{d q}\left(g_{t}, S g_{t}\right)+\mu_{3} \delta_{d q}\left(z^{*}, S z^{*}\right) \\
& +\mu_{4} \frac{\delta_{d q}\left(g_{t}, S g_{t}\right) . \delta_{d q}\left(z^{*}, S z^{*}\right)}{1+\delta_{d q}\left(g_{t}, z^{*}\right)} .
\end{aligned}
$$

Taking $t \rightarrow+\infty$, we have

$$
\delta_{d q}\left(z^{*}, S z^{*}\right)<\mu_{3} \delta_{d q}\left(z^{*}, S z^{*}\right),
$$




$$
\left(1-\mu_{3}\right) \delta_{d q}\left(z^{*}, S z^{*}\right)<0
$$

This is a contradiction, so $\delta_{d q}\left(z^{*}, S z^{*}\right)=0$, so $z^{*} \in S z^{*}$. Hence $z^{*}$ is a fixed point of $S$.

Example 2.2 Let $M=[0,+\infty)$. Consider $\delta_{d q}: M \times M \longrightarrow[0,+\infty)$ to be an $L$ - $R$-complete function weighted dislocated quasi-metric on $M$ defined as

$$
\delta_{d q}(g, w)=(2 g+3 w)^{2} .
$$

Obviously, $\delta_{d q}$ satisfies axiom $\left(\Delta_{1}\right)$. However, $\delta_{d q}$ is not symmetric, as $\delta_{d q}(1,2)=64 \neq 49=$ $\delta_{d q}(2,1)$. Define $S: M \times M \longrightarrow P(M)$ as $S(g)=\left[\frac{3 g}{10}, \frac{2 g}{3}\right]$. Take $\mu_{1}=\frac{1}{2}, \mu_{2}=\frac{1}{4}, \mu_{3}=\frac{1}{8}, \mu_{4}=\frac{1}{10}$, then $\mu_{1}+\mu_{2}+\mu_{3}+\mu_{4}<1$. Taking $\tau=0.2$ and $\mathcal{F}(g)=\ln g$, we have

$$
\begin{aligned}
\tau+ & \max \left\{\mathcal{F}\left(H_{\delta_{d q}}(S g, S w)\right), \mathcal{F}\left(H_{\delta_{d q}}(S w, S g)\right)\right\} \\
\leq & \min \left\{\mathcal{F}\left(\mu_{1} \delta_{d q}(g, w)+\mu_{2} \delta_{d q}(g, S g)+\mu_{3} \delta_{d q}(w, S w)+\mu_{4} \frac{\delta_{d q}(g, S g) \cdot \delta_{d q}(w, S w)}{1+\delta_{d q}(g, w)}\right),\right. \\
& \left.\mathcal{F}\left(\mu_{1} \delta_{d q}(w, g)+\mu_{2} \delta_{d q}(S g, g)+\mu_{3} \delta_{d q}(S w, w)+\mu_{4} \frac{\delta_{d q}(S g, g) \cdot \delta_{d q}(S w, w)}{1+\delta_{d q}(w, g)}\right)\right\} \\
= & \mathcal{F}\left(\mu_{1} \delta_{d q}(w, g)+\mu_{2} \delta_{d q}(S g, g)+\mu_{3} \delta_{d q}(S w, w)+\mu_{4} \frac{\delta_{d q}(S g, g) \cdot \delta_{d q}(S w, w)}{1+\delta_{d q}(w, g)}\right) \\
= & \ln \left(\frac{1}{2}(2 g+3 w)^{2}+\frac{1}{4}\left(\frac{3 g}{5}+3 g\right)^{2}+\frac{1}{8}\left(\frac{3 w}{5}+3 w\right)^{2}+\frac{1}{10} \frac{\left(\frac{3 g}{5}+3 g\right)^{2} \cdot\left(\frac{3 w}{5}+3 w\right)^{2}}{1+(2 g+3 w)^{2}}\right) .
\end{aligned}
$$

Since all the conditions of Theorem 2.1 are fulfilled and 0 is a fixed point of $S$.

Corollary 2.3 Suppose that $\left(M, \delta_{d q}\right)$ is an L-R-complete function weighted dislocated quasi-metric space with respect to $(h, C) \in \gamma \times[0,+\infty)$. Let $S: M \rightarrow P(M)$ be a multivalued mapping, $\mathcal{F}:(0,+\infty) \rightarrow \mathbb{R}$ be a strictly increasing mapping, $\tau>0, \mu_{1}, \mu_{3}, \mu_{4} \geq 0$, $\eta_{1}=\frac{\mu_{1}}{1-\mu_{3}-\mu_{4}}<1$ and $\eta_{2}=\frac{\mu_{1}+\mu_{3}}{1-\mu_{4}}<1$ such that

$$
\begin{aligned}
\tau+ & \max \left\{\mathcal{F}\left(H_{\delta_{d q}}(S g, S w)\right), \mathcal{F}\left(H_{\delta_{d q}}(S w, S g)\right)\right\} \\
\leq & \min \left\{\mathcal{F}\left(\mu_{1} \delta_{d q}(g, w)+\mu_{3} \delta_{d q}(w, S w)+\mu_{4} \frac{\delta_{d q}(g, S g) . \delta_{d q}(w, S w)}{1+\delta_{d q}(g, w)}\right)\right. \\
& \left.\mathcal{F}\left(\mu_{1} \delta_{d q}(w, g)+\mu_{3} \delta_{d q}(S w, w)+\mu_{4} \frac{\delta_{d q}(S g, g) \cdot \delta_{d q}(S w, w)}{1+\delta_{d q}(w, g)}\right)\right\}
\end{aligned}
$$

whenever $\min \left\{H_{\delta_{d q}}(S g, S w), H_{\delta_{d q}}(S w, S g)\right\}>0, g, w \in\left\{M S\left(g_{t}\right)\right\} \cup\left\{z^{*}\right\}$, where $\left\{M S\left(g_{t}\right)\right\} \rightarrow z^{*}$. Then $z^{*}$ is the fixed point of $S$.

Corollary 2.4 Suppose that $\left(M, \delta_{d q}\right)$ is an L-R-complete function weighted dislocated quasi-metric space with respect to $(h, C) \in \gamma \times[0,+\infty)$. Let $S: M \rightarrow P(M)$ be a multivalued mapping, $\mathcal{F}:(0,+\infty) \rightarrow \mathbb{R}$ be a strictly increasing mapping, $\tau>0, \mu_{1}, \mu_{2}, \mu_{4} \geq 0$, 


$$
\begin{aligned}
\eta_{1}=\frac{\mu_{1}+\mu_{2}}{1-\mu_{4}}<1 \text { and } \eta_{2}=\frac{\mu_{1}}{1-\mu_{2}-\mu_{4}}<1 \text { such that } \\
\tau+\max \left\{\mathcal{F}\left(H_{\delta_{d q}}(S g, S w)\right), \mathcal{F}\left(H_{\delta_{d q}}(S w, S g)\right)\right\} \\
\leq \min \left\{\mathcal{F}\left(\mu_{1} \delta_{d q}(g, w)+\mu_{2} \delta_{d q}(g, S g)+\mu_{4} \frac{\delta_{d q}(g, S g) . \delta_{d q}(w, S w)}{1+\delta_{d q}(g, w)}\right),\right. \\
\left.\mathcal{F}\left(\mu_{1} \delta_{d q}(w, g)+\mu_{2} \delta_{d q}(S g, g)+\mu_{4} \frac{\delta_{d q}(S g, g) \cdot \delta_{d q}(S w, w)}{1+\delta_{d q}(w, g)}\right)\right\}
\end{aligned}
$$

whenever $\min \left\{H_{\delta_{d q}}(S g, S w), H_{\delta_{d q}}(S w, S g)\right\}>0, g, w \in\left\{M S\left(g_{t}\right)\right\} \cup\left\{z^{*}\right\}$, where $\left\{M S\left(g_{t}\right)\right\} \rightarrow z^{*}$. Then $z^{*}$ is the fixed point of $S$.

Corollary 2.5 Suppose that $\left(M, \delta_{d q}\right)$ is an L-R-complete function weighted dislocated quasi-metric space with respect to $(h, C) \in \gamma \times[0,+\infty)$. Let $S: M \rightarrow P(M)$ be a multivalued mapping, $\mathcal{F}:(0,+\infty) \rightarrow \mathbb{R}$ be a strictly increasing mapping, $\tau>0, \mu_{1}, \mu_{2}, \mu_{3} \geq 0$, $\eta_{1}=\frac{\mu_{1}+\mu_{2}}{1-\mu_{3}}<1$ and $\eta_{2}=\frac{\mu_{1}+\mu_{3}}{1-\mu_{2}}<1$ such that

$$
\begin{aligned}
\tau+ & \max \left\{\mathcal{F}\left(H_{\delta_{d q}}(S g, S w)\right), \mathcal{F}\left(H_{\delta_{d q}}(S w, S g)\right)\right\} \\
\leq & \min \left\{\mathcal{F}\left(\mu_{1} \delta_{d q}(g, w)+\mu_{2} \delta_{d q}(g, S g)+\mu_{3} \delta_{d q}(w, S w)\right),\right. \\
& \left.\mathcal{F}\left(\mu_{1} \delta_{d q}(w, g)+\mu_{2} \delta_{d q}(S g, g)+\mu_{3} \delta_{d q}(S w, w)\right)\right\}
\end{aligned}
$$

whenever $\min \left\{H_{\delta_{d q}}(S g, S w), H_{\delta_{d q}}(S w, S g)\right\}>0, g, w \in\left\{M S\left(g_{t}\right)\right\} \cup\left\{z^{*}\right\}$, where $\left\{M S\left(g_{t}\right)\right\} \rightarrow z^{*}$. Then $z^{*}$ is the fixed point of $S$.

\section{Application}

In this section, we present our main result for single-valued mappings and investigate the uniqueness of the fixed point as well. An application is given to the obtained result.

Theorem 3.1 Suppose that $\left(M, \delta_{d q}\right)$ is an L-R-complete function weighted dislocated quasi-metric space with respect to $(h, C) \in \gamma \times[0,+\infty)$. Let $S: M \rightarrow M$ be a mapping, $\mathcal{F}:(0,+\infty) \rightarrow \mathbb{R}$ be a strictly increasing mapping, $\tau>0, \mu_{1}, \mu_{2}, \mu_{3}, \mu_{4} \geq 0, \eta_{1}=\frac{\mu_{1}+\mu_{2}}{1-\mu_{3}-\mu_{4}}<1$ and $\eta_{2}=\frac{\mu_{1}+\mu_{3}}{1-\mu_{2}-\mu_{4}}<1$ such that

$$
\begin{aligned}
\tau+ & \max \left\{\mathcal{F}\left(\delta_{d q}(S g, S w)\right), \mathcal{F}\left(\delta_{d q}(S w, S g)\right)\right\} \\
\leq & \min \left\{\mathcal{F}\left(\mu_{1} \delta_{d q}(g, w)+\mu_{2} \delta_{d q}(g, S g)+\mu_{3} \delta_{d q}(w, S w)+\mu_{4} \frac{\delta_{d q}(g, S g) . \delta_{d q}(w, S w)}{1+\delta_{d q}(g, w)}\right)\right. \\
& \left.\mathcal{F}\left(\mu_{1} \delta_{d q}(w, g)+\mu_{2} \delta_{d q}(S g, g)+\mu_{3} \delta_{d q}(S w, w)+\mu_{4} \frac{\delta_{d q}(S g, g) . \delta_{d q}(S w, w)}{1+\delta_{d q}(w, g)}\right)\right\}
\end{aligned}
$$

where, $g, w \in M$. Then there exists a unique fixed point of $S$.

Proof The proof of Theorem 3.1 is similar to the proof of Theorem 2.1. Here we prove only uniqueness. Suppose that $g^{*}$ and $w^{*}$ are the two distinct fixed points of $S$, then $\delta_{d q}\left(g^{*}, w^{*}\right)>$ 
0 . By inequality (3.1), we have

$$
\begin{aligned}
& \tau+\mathcal{F}\left(\delta_{d q}\left(g^{*}, w^{*}\right) \leq\right. \tau+\max \left\{\mathcal { F } \left(\delta_{d q}\left(S g^{*}, S w^{*}\right), \mathcal{F}\left(\delta_{d q}\left(S w^{*}, S g^{*}\right)\right\}\right.\right. \\
& \leq \mathcal{F}\left(\mu_{1} \delta_{d q}\left(g^{*}, w^{*}\right)+\mu_{2} \delta_{d q}\left(g^{*}, S g^{*}\right)+\mu_{3} \delta_{d q}\left(w^{*}, S w^{*}\right)\right. \\
&\left.+\mu_{4} \frac{\delta_{d q}\left(g^{*}, S g^{*}\right) . \delta_{d q}\left(w^{*}, S w^{*}\right)}{1+\delta_{d q}\left(g^{*}, w^{*}\right)}\right), \\
& \tau+\mathcal{F}\left(\delta_{d q}\left(g^{*}, w^{*}\right) \leq\right. \mathcal{F}\left(\mu_{1} \delta_{d q}\left(g^{*}, w^{*}\right)\right), \\
& \delta_{d q}\left(g^{*}, w^{*}\right)<\mu_{1} \delta_{d q}\left(g^{*}, w^{*}\right), \\
& \delta_{d q}\left(g^{*}, w^{*}\right)<\delta_{d q}\left(g^{*}, w^{*}\right) .
\end{aligned}
$$

As $\delta_{d q}\left(g^{*}, w^{*}\right)>0$, therefore a contradiction arises. So, we have $g^{*} \in M$, a unique fixed point of $S$.

Remark By taking a bi-complete function weighted quasi-metric space, $\mu_{2}=\mu_{3}=\mu_{4}=0$, $\tau>0$, and $\mathcal{F}(\alpha)=\ln (\alpha)$ in Theorem 3.1, we obtain the result of Karapınar et al. [17] as follows.

Corollary 3.2 Let $\left(M, \delta_{q}\right)$ be a bi-complete function weighted quasi-metric space and $S$ be a mapping from $M$ to $M$. Suppose that there exists $k=\mu_{1} e^{-\tau} \in(0,1)$ such that

$$
\delta_{q}(S g, S w) \leq k \delta_{q}(g, w), \quad g, w \in M
$$

Then S possesses a unique fixed point $g \in M$.

Remark By taking a bi-complete function weighted quasi-metric space, $\mu_{1}=\mu_{4}=0$ and $\mu_{2}=\mu_{3}, \tau>0$ and $\mathcal{F}(\alpha)=\ln (\alpha)$ in Theorem 3.1, we obtain the result of Karapinar et al. [17] as follows.

Corollary 3.3 Let $\left(M, \delta_{q}\right)$ be a bi-complete function weighted quasi-metric space and $S$ be a mapping from $M$ to $M$. Suppose that there exists $\mu=\mu_{2} e^{-\tau} \in(0,1 / 2)$ such that

$$
\delta_{q}(S g, S w) \leq \mu\left[\delta_{q}(g, S g)+\delta_{q}(w, S w)\right], \quad g, w \in M .
$$

Then $S$ possesses a unique fixed point $g \in M$.

Now we discuss the solution of Volterra type integral equation which is an application of Theorem 3.1. Consider the equation

$$
m(r)=\int_{0}^{r} H(r, q, m(q)) d q
$$

for all $r, q \in[0,1]$. For solution of (3.4), we follow the following process.

Let $M$ be a collection of all real-valued continuous functions on $[0,1]$ endowed with the $L-R$-complete function weighted dislocated quasi-metric space. Define the supremum 
norm as $\|m\|_{\tau}=\sup _{r \in[0,1]}\left\{|m(r)| e^{-\tau r}\right\}$ for $m \in M$, where $\tau>0$. Now, define

$$
\delta_{d q}^{\tau}(m, z)=\left[\sup _{r \in[0,1]}\left\{|2 m(r)+3 z(r)| e^{-\tau r}\right\}\right]^{2}=\|2 m+3 z\|_{\tau}^{2}
$$

for all $m, z \in M$, with these settings, $\left(M, \delta_{d q}^{\tau}\right)$ becomes an $L-R$-complete function weighted dislocated quasi-metric space.

Let us prove the theorem given as under to make sure the existence of solution of (3.4).

Theorem 3.4 Suppose that the following conditions are satisfied:

(i) $H:[0,1] \times[0,1] \times C\left([0,1], \mathbb{R}_{+}\right) \rightarrow \mathbb{R}_{+}$;

(ii) $S: M \rightarrow M$ is defined by

$$
\operatorname{Sm}(r)=\int_{0}^{r} H(r, q, m(q)) d q
$$

Suppose that $\tau>0$ exists, such that

$$
\max \{2 H(r, q, m)+3 H(r, q, z), 2 H(r, q, z)+3 H(r, q, m)\} \leq \frac{\tau N(m, z) e^{\tau q}}{\tau N(m, z)+1}
$$

for $m, z \in C\left([0,1], \mathbb{R}_{+}\right)$and for all $r, q \in[0,1]$, where

$$
\begin{aligned}
N(m, z)= & \mu_{1}\|2 m+3 z\|^{2}+\mu_{2}\|2 m+3 S m\|^{2}+\mu_{3}\|2 z+3 S z\|^{2} \\
& +\mu_{4} \frac{\|2 m+3 S m\|^{2} \cdot\|2 z+3 S z\|^{2}}{1+\|2 m+3 z\|^{2}}
\end{aligned}
$$

where $\tau, \mu_{1}, \mu_{2}, \mu_{3}, \mu_{4}>0$ and $\mu_{1}+\mu_{2}+\mu_{3}+\mu_{4}<1$. Then (3.4) has a unique solution.

Proof By supposition (ii)

$$
\begin{aligned}
& |\max \{2 S m+3 S z, 2 S z+3 S m\}| \\
& =\max \left\{\int_{0}^{r}(2 H(r, q, m)+3 H(r, q, z)) d q, \int_{0}^{r}(2 H(r, q, z)+3 H(r, q, m)) d q\right\} \\
& \quad<\int_{0}^{r} \frac{\tau N(m, z)}{\tau N(m, z)+1} e^{\tau q} d q \\
& =\frac{\tau N(m, z)}{\tau N(m, z)+1} \int_{0}^{r} e^{\tau q} d q, \quad<\frac{N(m, z) e^{\tau r}}{\tau N(m, z)+1}, \\
& |\max \{2 S m+3 S z, 2 S z+3 S m\}|<\frac{\tau N(m, z)\left(e^{\tau r}-1\right)}{(\tau N(m, z)+1) \tau} \\
& |\max \{2 S m+3 S z, 2 S z+3 S m\}| e^{-\tau r}<\frac{N(m, z)}{\tau N(m, z)+1}, \\
& \|\max \{2 S m+3 S z, 2 S z+3 S m\}\| \|_{\tau}<\frac{N(m, z)}{\tau N(m, z)+1} .
\end{aligned}
$$


This implies

$$
\frac{\tau N(m, z)+1}{N(m, z)}<\frac{1}{\|\max \{2 S m+3 S z, 2 S z+3 S m\}\|_{\tau}} .
$$

That is,

$$
\tau+\frac{1}{N(m, z)}<\frac{1}{\|\max \{2 S m+3 S z, 2 S z+3 S m\}\|_{\tau}} .
$$

This further implies

$$
\begin{aligned}
& \tau-\frac{1}{\|\max \{2 S m+3 S z, 2 S z+3 S m\}\|_{\tau}}<\frac{-1}{N(m, z)}, \\
& \tau+\max \left\{\frac{-1}{\|2 S m+3 S z\|}, \frac{-1}{\|2 S z+3 S m\|}\right\}<\frac{-1}{N(m, z)} .
\end{aligned}
$$

For $\mathcal{F}(z)=\frac{-1}{\sqrt{z}} ; z>0$ and $\delta_{d q}^{\tau}(m, z)=\|2 m+3 z\|_{\tau}^{2}$, the conditions of Theorem 3.1 are fulfilled. Hence the Volterra integral equation given in (3.4) has a unique solution.

\section{Conclusion}

The notion of a function weighted $L-R$-complete dislocated quasi-metric space has been introduced. The condition $\delta_{d q}(g, g)=0$ from function weighted quasi-metric space has been excluded. The concept of bi-completeness has been generalized by introducing the concept of $L$ - $R$-completeness. We have established fixed point results fulfilling generalized rational type F-contraction for a multivalued mapping in this new framework. We have presented results for single-valued mappings and have investigated the uniqueness of the fixed point as well. An application and an example have also been constructed.

\section{Acknowledgements}

The fourth author would like to thank Ministry of Education Malaysia and Universiti Kebangsaan Malaysia for their research support.

\section{Funding}

This work was supported by the Ministry of Education Malaysia through grant (FRGS/1/2019/STG06/UKM/01/3).

\section{Availability of data and materials}

All the data utilized in this article have been included, and the sources where they were adopted were cited accordingly.

\section{Competing interests}

The authors declare that they have no competing interests.

\section{Authors' contributions}

Each author equally contributed to this paper, read and approved the final manuscript.

\section{Author details}

${ }^{1}$ Department of Mathematics and Statistics, Riphah International University, I-14, 44000 Islamabad, Pakistan. ${ }^{2}$ Department of Mathematical Sciences, Faculty of Science and Technology, Universiti Kebangsaan Malaysia, 43600 Bangi, Selangor, Malaysia. ${ }^{3}$ Faculty of Mechanical Engineering, University of Belgrade, Kraljice Marije 16, 11120 Beograd 35, Serbia.

\section{Publisher's Note}

Springer Nature remains neutral with regard to jurisdictional claims in published maps and institutional affiliations. 


\section{References}

1. Agarwal, R., Karapınar, E., Roldan-López-de-Hierro, A.F.: Fixed point theorems in quasi-metric spaces and applications to multidimensional fixed point theorems on G-metric spaces. J. Nonlinear Convex Anal. 16(9), 1787-1816 (2015)

2. Al-Mazrooei, A.E., Ahmad, J.: Fixed point theorems for rational contractions in F-metric spaces. J. Math. Anal. 10(6), 79-86 (2019)

3. Almezel, S.A., Ahmad, J., Marino, G.: Fixed point theorems for generalized ( $\alpha \beta-\psi)$-contractions in F-metric spaces with applications. Mathematics 8, 584 (2020)

4. Alnaser, L.A., Lateef, D., Fouad, H.A., Ahmad, J.: Relation theoretic contraction results in F-metric spaces. J. Nonlinear Sci. Appl. 12(5), 337-344 (2019)

5. Ameer, E., Arshad, M.: Two new generalization for F-contraction on closed ball and fixed point theorem with application. J. Math. Ext. 11, 1-24 (2017)

6. Ameer, E., Huang, H., Nazam, M., Arshad, M.: Fixed point theorems for multivalued contractions with admissible mappings in partial b-metric spaces and application. UPB Sci. Bull. 81(2), 97-108 (2019)

7. Arshad, M., Khan, S.U., Ahmad, J.: Fixed point results for F-contractions involving some new rational expressions. JP J. Fixed Point Theory Appl. 11, 79-97 (2016)

8. Beg, I., Arshad, M., Shoaib, A.: Fixed point in a closed ball in ordered dislocated quasi metric space. Fixed Point Theory 16(2), 195-206 (2015)

9. Chen, C., Karapınar, E., Rakolevic, V.: Existence of periodic fixed point theorems in the setting of generalized quasi-metric spaces. J. Appl. Math. 2014, 1-8 (2014)

10. Hitzler, P.: Generalized metrics and topology in logic programming semantics. PhD thesis, School of Mathematics, Applied Mathematics and Statistics, National University Ireland, University College Cork (2001)

11. Hussain, N., Roshan, J.R., Paravench, V., Abbas, M.: Common fixed point results for weak contractive mappings in ordered dislocated $b$-metric space with applications. J. Inequal. Appl. 2013, 486 (2013)

12. Hussain, N., Salimi, P.: Suzuki-Wardowski type fixed point theorems for $\alpha$-GF-contractions. Taiwan. J. Math. 18(6), 1879-1895 (2014)

13. Jleli, M., Samet, B.: On a new generalization of metric spaces. J. Fixed Point Theory Appl. 20(128), 1-20 (2018)

14. Kadelburg, Z., Radenović, S.: Notes on some recent papers concerning F-contractions in b-metric spaces. Constr. Math. Anal. 1(2), 108-112 (2018)

15. Karapınar, E., Fulga, A., Agarwal, R.P.: A survey: F-contractions with related fixed point results. J. Fixed Point Theory Appl. 22, 69 (2020)

16. Karapınar, E., Gholizadeh, L., Alsulami, H.H., Noorwali, M.: $\alpha-(\psi, \varphi)$ contractive mappings on quasi-partial metric spaces. Fixed Point Theory Appl. 2015, 105 (2015)

17. Karapınar, E., Pitea, A., Shatanawi, W.: Function weighted quasi-metric spaces and fixed point results. IEEE Access 7 , 89026-89032 (2019)

18. Karapınar, E., Roldan-López-de-Hierro, A., Samet, B.: Matkowski theorems in the context of quasi-metric spaces and consequences on G-metric spaces. An. Ştiinţ. Univ. 'Ovidius' Constanţa, Ser. Mat. 24(1), 309-333 (2016)

19. Karapınar, E., Romaguera, S.: On the weak form of Ekeland's variational principle in quasi-metric spaces. Topol. Appl. $184,54-60(2015)$

20. Karapınar, E., Romaguera, S., Tirado, P.: Contractive multivalued maps in terms of Q-functions on complete quasimetric spaces. Fixed Point Theory Appl. 2014, 53 (2014)

21. Karapınar, E., Salimi, P.: Dislocated metric space to metric spaces with some fixed point theorems. Fixed Point Theory Appl. 2013, 1 (2013)

22. Lateef, D., Ahmad, J.: Dass and Gupta's fixed point theorem in F-metric spaces. J. Nonlinear Sci. Appl. 12, 405-411 (2019)

23. Mahmood, Q., Shoaib, A., Rasham, T., Arshad, M.: Fixed point results for the family of multivalued F-contractive mappings on closed ball in complete dislocated b-metric spaces. Mathematics 7(1), 56 (2019)

24. Nadler, S.: Multivalued contraction mappings. Pac. J. Math. 30, 475-488 (1969)

25. Nazam, M., Acar, O.: Fixed points of $(\alpha, \psi)$-contractions in Hausdorff partial metric spaces. Math. Methods Appl. Sci. 42(16), 5159-5173 (2019)

26. Nazam, M., Aydi, H., Noorani, M.S.: Existence of fixed points of four maps for a new generalized F-contraction and an application. J. Funct. Spaces 2019, 1-8 (2019)

27. Nazam, M., Hussain, N., Hussain, A., Arshad, M.: Fixed point theorems for weakly admissible pair of F-contractions with application. Nonlinear Anal., Model. Control 24(6), 898-918 (2019)

28. Noorwali, M., Alsulami, H.H., Karapınar, E.: Some extensions of fixed point results over quasi-JS-spaces. J. Funct. Spaces 2016, 1-8 (2016)

29. Panda, S.K., Abdeljawad, T., Ravichandran, C.: Novel fixed point approach to Atangana-Baleanu fractional and Lp-Fredholm integral equations. Alex. Eng. J. 59(4), 1959-1970 (2020)

30. Rasham, T., Shoaib, A., Hussain, N., Arshad, M., Khan, S.U.: Common fixed point results for new Ćirić-type rational multivalued F-contraction with an application. J. Fixed Point Theory Appl. 20(1), 1-16 (2018)

31. Roldán-López-de-Hierro, A., Karapınar, E., de la Sen, M.: Coincidence point theorems in quasi-metric spaces without assuming the mixed monotone property and consequences in G-metric spaces. Fixed Point Theory Appl. 2014, 184 (2014)

32. Sgroi, M., Vetro, C.: Multi-valued F-contractions and the solution of certain functional and integral equations. Filomat 27(7), 1259-1268 (2017)

33. Shoaib, A., Alshoraify, S.S., Arshad, M.: Fixed point results for a pair of multivalued mappings in quasi metric spaces via new approach. UPB Sci. Bull., Ser. A 82(1), 121-132 (2020)

34. Shoaib, A., Kazi, S., Tassaddiq, A., Alshoraify, S.S., Rasham, T.: Double controlled quasi-metric type spaces and some results. Complexity 2020, 1-8 (2020)

35. Shoaib, A., Khan, I.S., Hassan, Z:: Generalized contraction involving an open ball and common fixed point of multivalued mappings in ordered dislocated quasi metric spaces. Filomat 34(2), 323-338 (2020)

36. Shoaib, A., Kumam, P., Alshoraify, S., Arshad, M.: Fixed point results in double controlled quasi metric type spaces. AIMS Math. 6(2), 1851-1864 (2021) 
37. Shoaib, A., Rasham, T., Rawashdy, A., Arshad, M.: DQF-contraction and related fixed point results in DQM spaces with application. J. Math. Anal. 10(6), 32-45 (2015)

38. Wardowski, D.: Fixed point theory of a new type of contractive mappings in complete metric spaces. Fixed Point Theory Appl. 2012, 1 (2012)

39. Wilson, W.A.: On quasi metric spaces. Am. J. Math. 53, 675-684 (1931)

Submit your manuscript to a SpringerOpen ${ }^{\circ}$ journal and benefit from:

- Convenient online submission

$\checkmark$ Rigorous peer review

- Open access: articles freely available online

- High visibility within the field

Retaining the copyright to your article

Submit your next manuscript at $\gg$ springeropen.com 